\title{
ASSESSMENT OF DEGREE OF HEARING LOSS DEPENDING ON SITE AND SIZE OF TYMPANIC MEMBRANE PERFORATION IN INACTIVE MUCOSAL VARIETY OF CHRONIC OTITIS MEDIA
}

\author{
Shyama Bandyopadhyay1, Anindita Sengupta², Arya Brata Dubev³, Arunabha Sengupta ${ }^{4}$ \\ 1Medical Officer Specialist, Department of ENT and Head Neck Surgery, Institute of Postgraduate Medical Education and Research, \\ Kolkata, West Bengal, India. \\ ${ }^{2}$ Residential Medical Officer, Department of ENT and Head Neck Surgery, Institute of Postgraduate Medical Education and Research, \\ Kolkata, West Bengal, India. \\ 3Junior Resident, Department of ENT and Head Neck Surgery, Institute of Postgraduate Medical Education and Research, Kolkata, \\ West Bengal, India. \\ ${ }^{4}$ Professor and HOD, Department of ENT and Head Neck Surgery, Institute of Postgraduate Medical Education and Research, Kolkata, \\ West Bengal, India.
}

\begin{tabular}{l}
\hline ABSTRACT \\
BACKGROUND \\
Tympanic membrane perforation is one of the most common causes of hearing impairment. We have compared the audiological \\
results depending on the site and size of tympanic membrane perforation.
\end{tabular}

\section{METHODS}

This is a prospective study of 120 patients who attended ENT OPD of our department during the period of April 2012 to March 2013 with inactive mucosal variety of chronic otitis media. All 120 patients had undergone video otoendoscopic evaluation and pure tone audiometry to evaluate the size and site of perforation and correlate it with AB gap and AC threshold.

\section{RESULTS}

In our study, pin-point perforation has an average of $28 \mathrm{~dB}$ (SD-3.79) AC threshold and 13.94 Db (SD-3.43) AB gap while subtotal perforation has $47.36 \mathrm{~dB}$ AC thresholds and $34.64 \mathrm{~dB}$ AB gap. Small, medium and large size perforations have an average of 35.25 $\mathrm{dB}, 39.93 \mathrm{~dB}, 44.77 \mathrm{~dB}$ AC threshold and $20.79 \mathrm{~dB}, 26.13 \mathrm{~dB}$ and $30.31 \mathrm{~dB}$ AB gap respectively. The results are statistically significant $(\mathrm{p}<0.001)$. Overall, central type of tympanic membrane perforation has highest (Mean $A C$ threshold $43.26 \mathrm{~dB}$ and $A B$ gap $29.28 \mathrm{~dB}$ ) hearing loss compared to single quadrant perforation. Posterior perforation has mean $A C$ threshold of $36 \mathrm{~dB}, A B$ gap $21.35 \mathrm{~dB}$ while anterior and inferior perforations have mean $\mathrm{AC}$ threshold $27.50 \mathrm{~dB}$ and $28.86 \mathrm{~dB}$ and mean $\mathrm{AB}$ gap $14 \mathrm{~dB}$ and 15.5 $\mathrm{dB}$ respectively.

\section{CONCLUSIONS}

As the size of perforation increases, the hearing loss increases, and it is also clear that posterior perforation has more hearing loss compared to anterior perforation. The results are statistically significant.

\section{KEY WORDS}

Chronic Otitis Media, Central Perforation, Audiology

HOW TO CITE THIS ARTICLE: Bandyopadhyay S, Sengupta A, Dubev AB, et al. Assessment of degree of hearing loss depending on site and size of tympanic membrane perforation in inactive mucosal variety of chronic otitis media. J. Evolution Med. Dent. Sci. 2019;8(27):2176-2180, DOI: $10.14260 /$ jemds/2019/477

\section{BACKGROUND}

Tympanic membrane (TM) perforation is one of the most common causes of hearing impairment. Infection, acute or chronic is the principle cause of TM perforation. Majority of post-acute infection and traumatic TM perforations however heal spontaneously or with conservative treatment. Chronic otitis media is an inflammatory condition of the middle ear cleft.1,2 The tympanic membrane (TM) serves as a key component of the tympano-ossicular system for sound transmission.

'Financial or Other Competing Interest': None.

Submission 09-05-2019, Peer Review 22-06-2019,

Acceptance 28-06-2019, Published 08-07-2019.

Corresponding Author:

Anindita Sengupta,

244 AJC Bose Road

Department of ENT and Head Neck Surgery,

Institute of Postgraduate Medical Education and Research,

SSKM Hospital, Kolkata, West Bengal, India.

E-mail: anindita122@gmail.com

DOI: $10.14260 /$ jemds $/ 2019 / 477$

\section{(c) $(\mathbf{P}) \ominus$}

Apart from conduction of sound waves across the middle ear, tympanic membrane also sub-serves a protective function to the middle ear cleft and round window niche. Intact tympanic membrane protects the middle ear cleft from infections and shields the round window from direct sound waves which is referred to as round window baffle ${ }^{1}$. This shield is necessary to create a phase differential so that the sound wave does not impact on the oval and round windows simultaneously. ${ }^{3}$ The aim of the study was to assess the degree of conductive hearing loss in relation to sites of tympanic membrane perforation in inactive mucosal variety of chronic otitis media and to determine the degree of conductive hearing loss related to different sizes of tympanic membrane perforation. It is generally believed that the larger the perforation on the tympanic membrane, the greater the decibel loss in sound perception. A total absence of the tympanic membrane would lead to a loss in the transformer action of the middle ear. Similarly, the location of the perforation is also considered to have significant impact on the magnitude of hearing loss. However, some workers believe that there is no significant effect associated with size 
or location of the perforation on the hearing loss. Thus, there have been various opinions about the size and site of TM perforation having different effects on the hearing status of the patient. However, further work and evidence is required to understand the physiology of sound conduction and status of hearing loss in cases of TM perforation. There have been few systematic studies of the structural features determining the magnitude of the resulting conductive hearing loss in cases of tympanic membrane perforation. This study is carried out to investigate the relationship between the effect of site and size of tympanic membrane perforation on hearing loss in inactive mucosal type of chronic otitis media with a view to contributing to the body of knowledge on this subject.

\section{METHODS}

The present study was carried out in the department of E.N.T. of our Institute.

\section{Ethical Clearance}

All procedures performed in studies involving human participants were in accordance with ethical standards of the institutional and/or national research committee and with the 1964 Helsinki declaration and its later amendments or comparable ethical standards. Informed consent was obtained from all individual participants included in the study. Patients were selected ranging from 16 to 60 years who has dry CP for at least 6 weeks. Perforation with preexisting or congenital hearing loss, patients with sensoryneural hearing loss, patients with traumatic perforation and residual perforation in acute otitis media and patients with squamosal disease were excluded from our study. So, patients fulfilling the above criteria who came between March 2012 to April 2013 constitute 120 patients. All patients were subjected to detailed history taking, clinical examination including otoscopy, otoendoscopy and microscopic examination and investigations including Pure Tone Audiometry (PTA). Findings were recorded in proforma prepared at the outset.

\section{Determination of Size and Site of Perforation}

Video Otoendoscopy of all cases with Storz 0-degree endoscope were done. All images were recorded on the computer [DELL INSPIRON N5050]. Using 'Image J' [version 1.35j of Wayne Rasband, National Institute of Health U.S.A.] geometrical package, the area of perforation $(\mathrm{P})$ and the entire area of the tympanic membrane $(\mathrm{T})$ were calculated. Then, the percentage area of the perforation [P/T X 100\%] for each ear was obtained. For the purpose of this study, the tympanic membrane was comprehensively divided into four segments for clarity (1-3 represents the three quadrants and the 4 represents involvement of more than one quadrant.), (1) Anterior; (2) Posterior; (3) Inferior and (4) Central for the localization of the site of perforation.

\section{Audiometry}

PTA was done by amplaid 460 model, audiometer in a sound proof room by the same audiologist. The patients' hearing levels in decibel $(\mathrm{dB})$ were assessed at frequencies $0.25 \mathrm{KHz}$, $0.5 \mathrm{KHz}, 1 \mathrm{KHz}, 2 \mathrm{KHz}, \& 4 \mathrm{KHz}$ respectively in an acoustically treated sound proof room. Air and bone conduction threshold were determined. The mean hearing loss was calculated through the pure tone average taken at $0.5 \mathrm{KHz}, 1 \mathrm{KHz}, 2 \mathrm{KHz}$, $\& 4 \mathrm{KHz}$ for each case.

\section{RESULTS}

For statistical analysis 120 patients were divided into five groups according to their size of perforation; Pinpoint $(<$ or $=$ $20 \%)$, Small (21-40\%), Medium (41-60\%), Large (61-80\%), Subtotal $(>80 \%)$ \& into four groups according to their site of perforation; Anterior (A), Posterior (P), Inferior (I), Central (Cn). Here central denotes involvement of more than one quadrant. Results were analysed according to these groups. One-way ANOVA, Pearson's chi square test, t-test \& correlation study were used wherever applicable to evaluate results.

\section{Air Conduction (AC) Threshold}

The four frequency mean Air Conduction (AC) threshold was within the range of $25-51 \mathrm{~dB} ; \&$ the overall mean value was $39.32 \mathrm{~dB}$. The mean AC threshold among the size \& site groups were statistically significant ( $\mathrm{p}$ value- $<0.001$ ). As size increases AC threshold increases.

\section{Air Bone (A-B) Gap}

In my study the mean $A-B$ gap was within the range of 10-40 $\mathrm{dB}$; \& overall mean value was $25.33 \mathrm{~dB}$. The mean A-B gap among the Size \& site groups were statistically significant ( $p$ value- $<0.001$ ).As size increases A-B gap increases.

\begin{tabular}{|c|c|c|c|c|c|}
\hline Size Groups & Mean & N & Std. Deviation & Minimum & Maximum \\
\hline$<20 \%$ (pin point) & 28.00 & 16 & 3.79 & 25 & 34 \\
\hline $21-40 \%$ (small) & 35.25 & 24 & 3.19 & 30 & 42 \\
\hline $\begin{array}{c}41-60 \% \\
\text { (medium) }\end{array}$ & 39.93 & 40 & 4.69 & 34 & 48 \\
\hline $61-80 \%$ (large) & 44.77 & 26 & 3.60 & 40 & 51 \\
\hline$>80 \%$ (subtotal) & 47.36 & 14 & 2.92 & 42 & 51 \\
\hline Total & $\mathbf{3 9 . 3 2}$ & $\mathbf{1 2 0}$ & $\mathbf{7 . 0 3}$ & $\mathbf{2 5}$ & $\mathbf{5 1}$ \\
\hline & Table 1. AC Threshold According to Size of Perforation \\
\hline p Value < 0.001 (One-way ANOVA)
\end{tabular}

\begin{tabular}{|c|c|c|c|c|c|}
\hline Size Groups & Mean & $\mathbf{N}$ & Std. Deviation & Minimum & Maximum \\
\hline$<20 \%$ (pin-point) & 13.94 & 16 & 3.43 & 10 & 20 \\
\hline $21-40 \%$ (small) & 20.79 & 24 & 3.08 & 15 & 25 \\
\hline $41-60 \%$ (medium) & 26.13 & 40 & 4.22 & 19 & 34 \\
\hline $61-80 \%$ (large) & 30.31 & 26 & 4.03 & 25 & 38 \\
\hline$>80 \%$ (subtotal) & 34.64 & 14 & 3.05 & 30 & 40 \\
\hline Total & 25.33 & 120 & 7.14 & 10 & 40 \\
\hline \multicolumn{6}{|c|}{ Table 2. Air-Bone Gap According to Size of Perforation } \\
\hline .001 (One & ANC & & & & \\
\hline
\end{tabular}

\begin{tabular}{|c|c|c|c|c|c|}
\hline Site Groups & Mean & N & Std. Deviation & Minimum & Maximum \\
\hline Anterior(A) & 27.50 & 8 & 2.67 & 25 & 30 \\
\hline Posterior(P) & 36.00 & 20 & 2.81 & 32 & 42 \\
\hline Inferior(I) & 28.86 & 14 & 3.25 & 25 & 34 \\
\hline Central(Cn) & 43.26 & 78 & 4.36 & 34 & 51 \\
\hline Total & 39.32 & 120 & 7.03 & 25 & 51 \\
\hline \multicolumn{7}{|c|}{ Table . AC Threshold According to Site of Perforation } \\
\hline p Value <0.001 (One-way ANOVA)
\end{tabular}

\begin{tabular}{|l|l|c|c|c|c|}
\hline Site Groups & Mean & N & Std. Deviation & Minimum & Maximum \\
\hline Anterior (A) & 14.00 & 8 & 4.21 & 10 & 15 \\
\hline Posterior (P) & 21.35 & 20 & 2.43 & 18 & 25 \\
\hline Inferior (I) & 15.50 & 14 & 3.23 & 12 & 20 \\
\hline Central (Cn) & 29.28 & 78 & 4.81 & 19 & 40 \\
\hline Total & 25.33 & 120 & 7.14 & 10 & 40 \\
\hline \multicolumn{6}{|c|}{ Table 4. Air Bone Gap According to Site of Perforation } \\
\hline p Value <0.001 (One-way ANOVA)
\end{tabular}



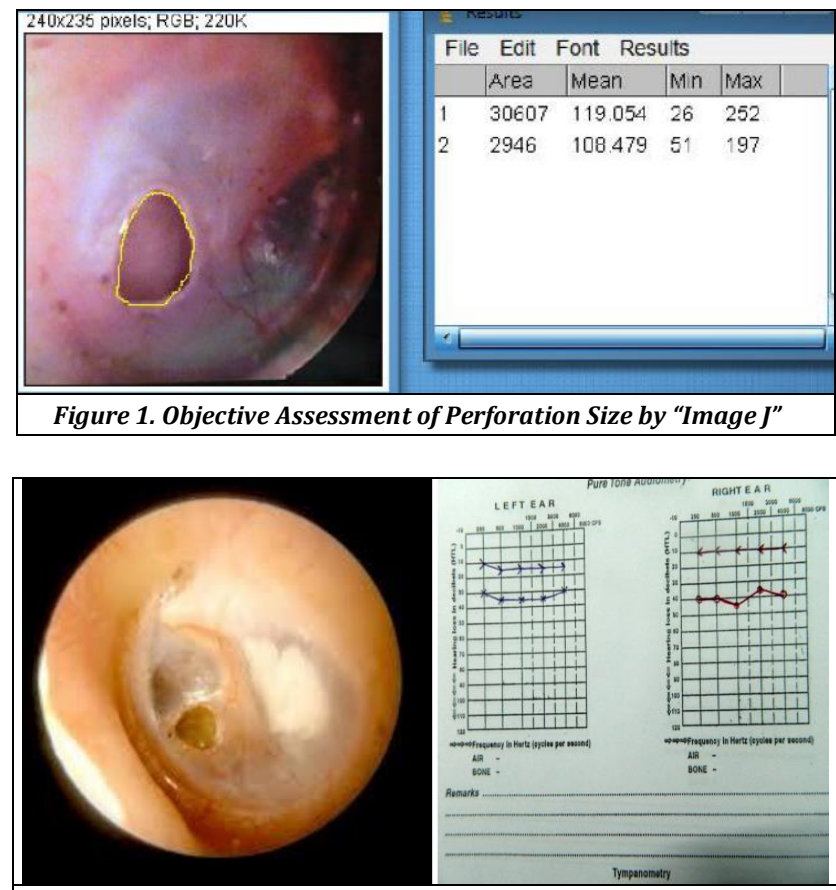

Figure 2. Anterior (A) Small Size Perforation and the Corresponding Pure Tone Audiometry

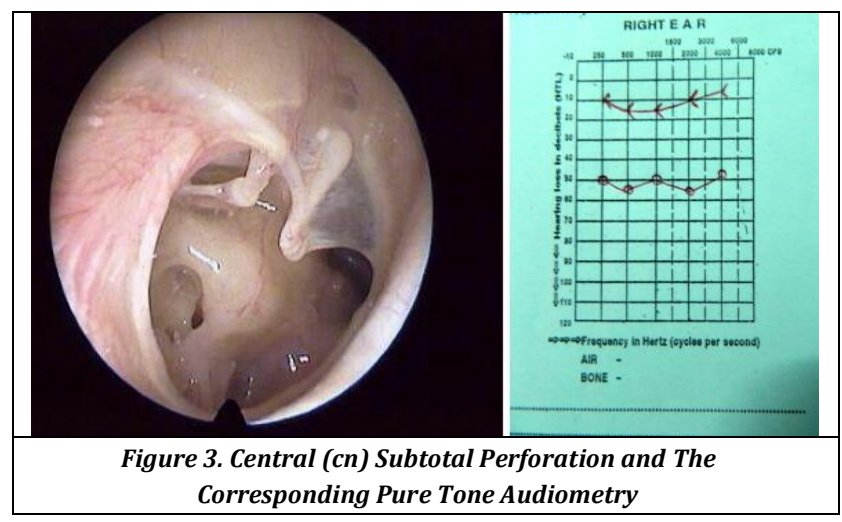

Posterior perforation has more A-C threshold among single quadrant perforations, however central perforation has maximum AC threshold ( $p$ value significant). Among anterior, posterior and inferior perforation, inferior perforation has maximum ABgap, however central has maximal $A B$ gap among all ( $p$ value significant).

In our study $52(43.33 \%)$ patients have right ear perforation and $68(56.67 \%)$ patients have left ear perforation whereas $53.33 \%(n=64)$ patients were male \& $46.67 \%(n=56)$ were female. Male: Female ratio was $1.14: 1$. It was found that sex distribution did not have any statistical significance among the size \& site groups. Age criteria was put between 16-60 years because younger children may not be able to understand the instructions during hearing assessment and in older group, presbycusis itself may affect the exact assessment of hearing loss, hence excluded in the study. There is no statistical significance between age distribution and site and size groups. We found that mean duration of symptoms like history of discharge per year, hearing loss, earache etc. in our study was 12.38 months. Shortest duration was 2 months \& the longest duration was 3 years. Duration of symptoms among the groups failed to achieve statistical significance. We found that the commonest symptom was hearing loss alone, found in $36.7 \% \quad(n=44)$ cases. Other symptoms were history of discharge per ear $26.7 \%(n=32)$; history of discharge with hearing loss $26.7 \%$ $(n=32) \&$ others symptoms e.g. Earache, tinnitus, were $13.3 \%$ $(n=16)$.

\section{DISCUSSION}

The age of patients in my study ranged from $16-60$ years, the mean age of presentation being 28.25 years. The reason for more number of patients in this age group may be attributed to the patients becoming more cautious socially about their hearing at this age and because of professional necessities or due to marriageable age group. Juvekar MR et $\mathrm{al}^{3}$ in his study took age range of 15-60 years. So, our study correlated with this study so far as age is concerned. Age range was 17-40 years in the study done by Zulkifal Awan et al (2008). 4 Titus $S$ Ibekwe et al (2009) ${ }^{1}$ took age range of 16-75 years and mean age of 35.4 years. From the above discussion it is clear that TM perforation can occur in any age group, but it is predominant in active age group that is within 21-30 years. In our study of 120 patients 64 patients were males and 56 were females. Male to female ratio was 1.14:1. The presentation in male patients slightly outnumbers the female. This could be due to the male sex being more aware of their disease and the incapacity produced because of the disease, as they are the working members of our society. Male: female ratio was 3:2 in a study done by Ashfaque Ahmed Shaikh et al. ${ }^{5}$ In another study by Ajmal Hussain et al, 6 the male: female ratio was 35:25. Shrestha $\mathrm{S}$, Sinha $\mathrm{BK}$ et al, ${ }^{7}$ had male: female ratio of 25:18. In our study Left ear was involved in $56.7 \%$ $(n=68)$ cases \& Right ear was involved in $43.3 \%(n=52)$ cases. Right to Left ratio was 1:1.3. In the study by Fadl A Fadl et al, 8 the ratio of right: left was 48:49 i.e. left side was more predominant than the right side. In a study by Edgar Sirena et al (2010), ${ }^{9}$ left: right ratio was 2: 1 . In a study by Francisco J. Aviles Jurado et al (2008) perforation was present in the right ear of $59 \%$ patients and in left ear of $41 \%$ patients. However, in a study by Adnan Salem Umar et al, ${ }^{10}$ the right: left ratio was 47:38. In our study, the commonest symptom was hearing loss alone, which was found in $36.7 \%(n=22)$ cases. Other symptoms were history of discharge from ear $26.7 \%$ $(n=16)$; history of discharge with hearing loss26.7 \%( $n=16)$ and other symptoms eg. Earache, tinnitus etc were $13.3 \%$ $(\mathrm{n}=8)$.

Sheahan P et al.11 in study showed $74 \%$ and $69 \%$ patients of tympanic membrane perforation had hearing loss and discharge from the ear. In our study the size of perforation was divided into five groups; Pinpoint $(<$ or $=20 \%)$, Small (21-40\%), Medium (41-60\%), Large (61-80\%), Subtotal $(>80 \%)$. The smallest size of perforation was $3 \%$ \& the largest was $94 \%$. The average size of perforation in my study was $48.9 \%$. Medium \& large size perforations are most prevalent in my study. In the study by Ashfaque Ahmed Shaikh et al..$^{5}$ medium size was $32 \%$, large was $48 \%$ and subtotal was $20 \%$ of total perforation. In a study by Sudhangshu Shekhar Biswas et al. (2010)12 medium size perforations were most common. In the comparative study by Zulkifal Awan et al (2008), 4 76.7\% cases had medium sized perforation. On the other hand, in the study of A Nepal et al. ${ }^{13}$ small perforations were the commonest. The mean AC threshold for pin point perforations was $28 \mathrm{~dB}$ but it was 47 $\mathrm{dB}$ for subtotal perforations, so mean $\mathrm{AC}$ depends on the size of perforation ( $p$ value $<0.001$ ).Austin et al.(5) reported that 
the larger the perforation, greater is the hearing impairment. Voss SE et al. ${ }^{14,15,16}$ in their various series in 2001 found that perforation size was the most important determinant of hearing loss. Berger et al (1997) ${ }^{17}$ \& Saeed and Ghamdi 18 found the same result in their study. In our study, if we consider the perforations involving only one quadrant the mean AC threshold for posterior perforations was $36 \mathrm{~dB}$ and it was $27 \mathrm{~dB}$ for anterior perforations. So, hearing loss is more for posterior perforations and it is statistically significant ( $\mathrm{p}$ value- $<0.001$ ). In the study by A Nepal et al. (2004), ${ }^{13}$ hearing loss was more in posterior perforations. Durko et al (1997) ${ }^{19}$ in their study found that hearing loss in posterior perforations to be up to $30 \mathrm{~dB}$. Berger et al (1997) ${ }^{18}$ \& Yung MW et al. (1995) ${ }^{20}$ found the same result in their study. The mean A-B gap among the size and site groups is statistically significant ( $p$ value- <0.001). Austin (1978) reported the above in his study about sound conduction of the diseased ears. In the study, he had compared such findings with those of others and with the experimental animals. ${ }^{21}$ Perforation size was found to be most important determination of hearing loss by Voss SE et al in their various series in $2001 .{ }^{23} \mathrm{He}$ mentioned that the volume of middle ear space combined with the tympanic cavity and mastoid air volume is also an important parameter that determine the amount of hearing loss caused by perforation. Thus, decreased middle ear air space volume results in greater airbone gap. Berger et al in 1997 carried out a prospective study of hearing loss in 120 cases during a 6-year period. They also found that the severity of conductive hearing loss to be proportional to the size of perforation. ${ }^{17}$

In the present study, hearing loss is found to be directly proportional to the size of perforation, which is consistent with all above studies. In total, 14 cases were found to have subtotal perforation with conductive hearing loss of $47.36 \mathrm{~dB}$ on average, while 26 large, 40 medium, 24 small and 16 pinpoint perforations had $45 \mathrm{~dB}, 40 \mathrm{~dB}, 35 \mathrm{~dB}$ and $28 \mathrm{~dB}$ average hearing loss respectively in speech frequencies below $2000 \mathrm{~Hz}$. This can be explained by the larger perforations resulting in loss of more middle ear and mastoid volume and more chances of diminishing phase effect as the perforation size increases due to direct exposure of sound pressure to the round and oval windows.

However, Saeed and Ghadami in a series of 183 patients (1987-92) found 122 patients having large perforation with average conductive hearing loss of $25.3 \mathrm{~dB}, 21$ medium sized perforation with hearing loss of $19.2 \mathrm{~dB}$ and 40 small perforations with $11.35 \mathrm{~dB}$ hearing loss. ${ }^{18} 145$ cases of chronic suppurative otitis media with central perforations and intact, mobile ossicles were clinically analysed by Durko et al. ${ }^{19}$ Hearing loss in perforations involving posteroinferior quadrant was found to be up to $30 \mathrm{~dB}$ while in rest of the central perforations, an average of $20 \mathrm{~dB}$ conductive hearing loss was found. Berger et al, in the same year in his study of over 120 cases also found that of all the locations, perforations involving the posteroinferior quadrant of the eardrum were associated with largest a-b gap. ${ }^{17}$ Audiometric assessment revealed that none of the patients suffered ossicular chain damage. Likewise, the fact that posterior perforations have greater hearing loss than anterior ones was revealed by Yung MW (1983) in the study of 100 cases. ${ }^{22}$ Admed et al (1979) studied 70 cases with similar results. They also noted that marginal perforations having greater hearing loss than that of similar sized central perforations. ${ }^{23}$ In present study, out of 120 cases, 20 cases involved posterior quadrant perforation having $36 \mathrm{~dB}$ AC threshold and $21 \mathrm{~dB}$ A-B gap on average. While 8 cases involved anterior quadrant having $28 \mathrm{~dB}$ AC threshold and $14 \mathrm{~dB} A-\mathrm{B}$ gap on average. However, Vose et al (2001) in their study do not agree with the notion that the location of the perforation should influence the resulting hearing loss in contradiction to all previous studies. ${ }^{22}$ Their result is questionable because the perforation located in posteroinferior quadrant has been proved to result loss in sound transmission and magnitude and phase of the sound pressures acting at the oval window and round window in various previous studies. Hearing loss was found to be directly proportional to the size of perforation and was statistically significant. Conductive hearing loss in the study was found to range from negligible to $43 \mathrm{~dB}$. Overall, perforations involving posterior quadrant were found to have maximum hearing loss. The extent of hearing loss to such extent can be explained by one or more of the following effects: 1) Diminished surface of the tympanic membrane on which sound pressure is exerted, causing diminished excursions of ossicular chain. 2) Diminished middle ear and mastoid air volume. 3) Sound vibrations reaching both the windows without dampening effect of the intact tympanic membrane. The result was consistent with the study by Voss et al 15 and Mc. Ardle and friends. ${ }^{24}$

\section{CONCLUSIONS}

Male predominance was seen in inactive mucosal variety of chronic otitis media. There is no relation of duration of symptoms with either size or site of tympanic membrane perforation in chronic otitis media. The size or location of tympanic membrane perforation is not related to age or sex of the patient. Larger the size of tympanic membrane perforation, greater is the conductive type of hearing loss in chronic otitis media. Large central perforations involving more than one quadrant results in greater degree of loss in sound perception. The location of perforation on the tympanic membrane has a significant effect on hearing loss. Posteriorly placed tympanic membrane perforations have greater degree of loss compared to anteriorly placed perforations.

\section{REFERENCES}

[1] Ibekwe TS, Nwaorgu OG, Adeosun AA, et al. Assessment of the size of tympanic membrane perforations: a comparison of clinical estimation with video-otoscopic calculations. Ear Nose Throat J 2008;87(10):567-9.

[2] Olusanya BO. Hearing impairment prevention in developing countries: making things happen. Int J Paedtr Otorhinolaryngol 2000;55(3):167-71.

[3] Juvekar MR, Juvekar RV. The double breasting technique of tympanoplasty: a study of 200 cases. Indian Journal of Otology 1999;5(3):145-8.

[4] Awan Z, Bashir H, Hussain A. Myringoplasty: a comparative study of different graft materials and various surgical techniques. Ann Pak Inst Med Sci 2008;4(4):209-11. 
[5] Shaikh AA, Onali MAS, Shaikh SM, et al. Outcome of tympanoplasty Type- 1 by underlay technique. JLUMHS 2009;8(1):80-4.

[6] Jurado FJA, Gil JLM, Secall MT, et al. Miringoplastia: seguimiento auditivo $\mathrm{y}$ estudio de factores pronósticos. Acta Otorrinolaringol Esp 2009;60(3):169-75.

[7] Shrestha S, Sinha BK. Hearing results after myringoplasty. Kathmandu University Medical College Journal 2006;4(4):455-9.

[8] Fadl FA. Outcome of type-1 tympanoplasty. Saudi Medical Journal 2003;24(1):58-61.

[9] Sirena E, Carvalho B, Buschle M, et al. Timanoplastia myringoplasty type 1 and in residency surgical results and audiometric. Int Arch Otorhinolaryngol Sao Paulo - Brazil 2010;14(4):417-21.

[10] Umar AS, Ahmed J. Anatomical and functional outcome following type 1 tympanoplasty in chronic tubotympanic SOM, Military Hospital Rawalpindi. March 2008, Issue-01.

[11] Sheehan P, Dounelly M, Kane R, et al. Clinical features of newly presented case of CSOM. Journal of Laryngology and Otology 2011;115:962-6.

[12] Sudhangshu SB, Md. Anwar H, Md. Monjurul A. Hearing evaluation after myringoplasty. Bangladesh J Otorhinolaryngol 2010;16(1):23-8.

[13] Nepal A, Bhandary S, Mishra SC, et al. Assessment of quantitative hearing loss in relation to the morphology of central tympanic membrane perforations. 2004.

[14] Vose SE, Rosowski JJ, Merchant SN, et al. Middle-ear function with tympanic-membrane perforations. I.
Measurements and mechanisms. J Acoust Soc Am 2001;110(3 Pt 1):1432-44.

[15] Vose SE, Rosowski JJ, Merchant SN, et al. How do tympanic membrane perforations affect human middle-ear sound transmission? Acta Otolaryngol 2001;121(2):169-73.

[16] Vose SE, Rosowski JJ, Merchant SN, et al. Middle-ear function with tympanic-membrane perforations. II. A simple model. J Acoust Soc Am 2001;110(3 Pt 1):144552.

[17] Berger G, Finkelstein Y, Avraham S, et al. Pattern of hearing loss in non-explosive blast injury of the ear. J Laryngol Otol 1997:111(12):1137-41.

[18] Al-Ghamdi SA. Tympanoplasty: factors influencing surgical outcome. Ann Saudi Med 1994;14(6):483-5.

[19] Durko T, Latkowski B. Extrameatal myringoplasty in the treatment of tympanic membrane perforations. Otolaryngol Pol 1997;51:274-7.

[20] Yung MW. Myringoplasty for subtotal perforation. Clincal Otolaryngol Allied Sci 1995;20(3):241-5.

[21] Austin DF. Sound conduction of the diseased ear. J Laryngol Otol 1978;92(5):367-93.

[22] Yung MW. Myringoplasty: hearing gain in relation to perforation site. J Laryngol Otol 1983;97(1):11-7.

[23] Ahmad SW, Ramani GV. Hearing loss in perforations of tympanic membrane. J Laryngol Otol 1979;93(11):1091-8.

[24] McArdle FE, Tonndorf J. Perforations of tympanic membrane and their effects upon middle-ear transmission. Arch Klin Exp Nasen Kehlkopfheilkd 1968;192(2):145-62. 